\title{
Expression of DR5 and c-FLIP proteins as novel prognostic biomarkers for non-small cell lung cancer patients treated with surgical resection and chemotherapy
}

\author{
HONGMEI ZHENG, YUTING ZHANG, YUTING ZHAN, SILE LIU, \\ JUNMI LU, QIUYUAN WEN and SONGQING FAN
}

Department of Pathology, The Second Xiangya Hospital, Central South University, Changsha, Hunan 410011, P.R. China

Received April 29, 2019; Accepted September 12, 2019

DOI: $10.3892 / o r .2019 .7355$

\begin{abstract}
TRAIL-R2 (DR5), one of the death receptors, can activate the extrinsic apoptosis pathway, while cellular FLICE-inhibitory protein (c-FLIP) can inhibit this pathway. Both of them play important roles in the occurrence and development of most tumors. To date, there is no relevant report concerning the relationship between expression of DR5 and c-FLIP protein and clinicopathological/prognostic implications in patients with non-small cell lung cancer (NSCLC) treated with surgical resection and chemotherapy. Thus, the aim of the present study was to investigate the potential prognostic significance of DR5 and c-FLIP in NSCLC patients and their predictive roles in the chemotherapeutic response. In the present study, DR5 and c-FLIP were detected by immunohistochemistry (IHC) in tissue microarrays of NSCLC. The results showed that the expression levels of DR5 and c-FLIP were significantly higher in lung squamous cell carcinoma (SCC) and lung adenocarcinoma (ADC) tissues compared with levels noted in the non-cancerous control lung tissues (all $\mathrm{P}<0.05$ ). In addition, DR5 expression was significantly increased in lung $\mathrm{ADC}(\mathrm{P}<0.001)$, whereas, c-FLIP was higher in lung SCC $(\mathrm{P}<0.001)$ and smoker patients with clinical stage III $(\mathrm{P}=0.019, \mathrm{P}=0.016$, respectively). In addition, NSCLC patients with overexpression of DR5 and loss of c-FLIP expression exhibited a higher overall survival (OS) rate as determined by Kaplan-Meier analysis $(\mathrm{P}=0.029, \mathrm{P}=0.038$, respectively). Multivariate analysis confirmed that high expression of DR5 and loss of c-FLIP expression were independent favorable prognostic factors for NSCLC patients $(\mathrm{P}=0.016, \mathrm{P}=0.035$, respectively). In conclusion, overexpression of DR5 and loss of c-FLIP expression may serve as novel favorable prognostic
\end{abstract}

Correspondence to: Professor Songqing Fan, Department of Pathology, The Second Xiangya Hospital, Central South University, 139 Renmin Middle Road, Changsha, Hunan 410011, P.R. China E-mail: songqingfan@csu.edu.cn

Key words: non-small cell lung cancer, DR5, c-FLIP, apoptosis, prognosis, biomarker biomarkers for NSCLC patients treated with chemotherapy after radical resection and used as predictors for tumor response to chemotherapy drugs.

\section{Introduction}

Lung cancer is one of the major malignant tumors diagnosed both in men and women worldwide. Lung cancer is classified into two histological subtypes: small-cell lung cancer (SCLC) accounting for $15 \%$ and non-small cell lung cancer (NSCLC) accounting for $85 \%$, mainly including squamous cell carcinoma (SCC) and adenocarcinoma (ADC) (1-3). Postoperative adjuvant chemotherapy aims to eliminate residual cancer cells and increases the overall survival of patients with NSCLC. Platinum-based chemotherapy is used as first-line therapy for advanced lung cancer (4), but not all patients experience favorable outcome. The cytotoxicity of these drugs has been demonstrated to be mediated by apoptosis and a defect in apoptosis makes cancer cells resistant to gemcitabine and cisplatin chemotherapy $(5,6)$. Therefore, the identification of response markers is helpful in predicting the response to platinum-based chemotherapy, and it is important to identify new biomarkers for determining the risks of occurrence and progression and the prognosis of lung cancer.

Apoptosis is a common type of programmed cell death observed in various tissues and cell types, and is mediated by a set of cysteine proteases activated specifically in apoptotic cells (7). DR5 (TRAIL-R2), one of the death receptors (DRs), is a plasma membrane protein containing intracellular death domains which transmit the death signals into cells $(8,9)$. When tumor necrosis factor-related apoptosis-inducing ligand (TRAIL) binds to DR5 or DR4 (TRAIL-R1), the adaptor FADD (Fas-associated protein with death domain) and caspase- 8 are further recruited, together forming the death-inducing signal complex (DISC) and activating the apoptosis pathway (10-12). Overexpression of DR5 can induce apoptosis of human cancer cells. It has been shown that DR5 is emerging as an important regulator of drug-induced apoptosis in human cancers (5). c-FLIP [cellular FLICE (FADD-like IL-1 $\beta$-converting enzyme)-inhibitory protein] is an important inhibitor of apoptosis protein (13). It is reported that c-FLIP can inhibit the activation of caspase- 8 by inhibiting the binding of 
pro-caspase- 8 to DISC, thus blocking Fas-mediated apoptosis signal transduction and ultimately inhibiting apoptosis (14-16). It has been reported that c-FLIP is overexpressed in many types of malignant tumors and is significantly correlated with the poor prognosis of patients (17). Thus, c-FLIP is a promising anticancer target (13).

Currently, more and more studies have shown that inhibition of tumor cell apoptosis is a marker of the occurrence and development of most or all types of tumors (18) and there is no report concerning whether or not the expression levels of DR5 and c-FLIP protein are associated with the clinicopathological features of NSCLC patients and the prognostic implication in these patients treated with chemotherapy after radical resection. Moreover, the association between the expression of DR5 and c-FLIP protein in NSCLC remains unclear. Therefore, in the present study, DR5 and c-FLIP protein were detected by IHC in tissue microarrays (TMA), including 227 cases of NSCLC and 49 cases of non-cancerous control lung tissue. Thus, the aim of the present study was to investigate potential prognostic significance of DR5 and c-FLIP in NSCLC patients and their predictive roles in the response of chemotherapy.

\section{Materials and methods}

Ethics statement. Samples were collected with informed consent and all protocols were approved by The Second Xiangya Hospital of Central South University Ethics Review Board (Scientific and Research Ethics Committee, no. S039/2011). All patients involved in the study or their guardians signed a written informed consent.

Clinical data and tissue microarrays (TMA). In this study, a total of 227 NSCLC patients aged from 25 to 78 years (mean age, 55.8 years), including 124 cases of ADC and 103 cases of SCC, and 49 cases of control normal lung specimens were obtained from the Second Xiangya Hospital of Central South University (Changsha, China) between January 2005 to January 2011. Complete clinical and follow-up data (Table SI) was available for all patients with written informed consent and this study was approved by the Ethics Committee of the Second Xiangya Hospital of Central South University (No: S039/2011). In this study, we chose the cutoff of age $(<56, \geq 56)$ based on the average age of these patients. Lymph nodes of the 'LNM status' mainly refer to the lymph nodes in the specimens of lobectomy $+\mathrm{LN}$ dissection. Whether the lymph nodes present with metastasis (LNM status) or not depended mainly on the results of the pathological diagnosis, and the classification of lymph nodes (N1, N2 and N3) has been included in the clinical stage. Duration of follow-up (median) was 1 to 60 months (27 months). For all patients a pathological diagnosis was confirmed according to the WHO histological classification of Lung Cancer (19) and the staging classification was carried out based on the Eighth Edition Lung Cancer Stage Classification (20). No patients had previously been treated with radiotherapy, cytotoxic chemotherapy, immunotherapy or target agents at the time of original operation, but all had adjuvant platinum doublet chemotherapy after lobectomy $+\mathrm{LN}$ dissection, including cisplatin/carboplatin + gemcitabine, cisplatin/carboplatin + paclitaxel/docetaxel and cisplatin/carboplatin + pemetrexed within the time period investigated. Tissue microarrays were constructed according to previously described technology $(21,22)$.

Immunohistochemistry and scores. The staining for samples on the TMAs was carried out with the ready-to-use Dako Envision $\mathrm{TM}^{+}$Dual Link System-HRP methods (Agilent Technologies, Inc.). As described in detail previously $(23,24)$, the staining condition for each antibody was adjusted according to the laboratory experience. A 1:4,000 dilution of the primary antibody to DR5: Apo2/TRAIL-R2/TRICK2/KILLER (rabbit polyclonal antibody, SKU 2019, ProSci) and a 1:300 dilution of the primary antibody to FLIP $_{S / L}$ (rabbit polyclonal antibody, catalog no. SC-8347; Santa Cruz Biotechnology, Inc.) were applied to assess the expression of these two proteins. In addition to the internal positive control, each experiment included positive control slides. A matched IgG isotype antibody was used as a negative control to confirm the specificity of the antibody. Immunohistochemical staining of TMA sections was independently evaluated by SF and JF, who were blinded to the clinicopathological data, under x200 magnification light microscopy. The evaluation was based on a semiquantitative method $(23,24)$ as follows: Overall score=percentage score $\mathrm{x}$ intensity score. Staining intensity for DR5 and c-FLIP was scored as 0 (negative), 1 (weak), 2 (moderate), and 3 (strong) and the extent of staining was scored as $0(0 \%), 1(1-25 \%)$, $2(26-50 \%), 3(51-75 \%)$, and 4 (76-100\%), depending on the percentage of positively stained cells. The scores ranged from 0 to 12 and the optimal cut-off levels for DR5 and c-FLIP were 7 and 4, respectively, according to the basis of log-rank test with respect to overall survival (OS) rates. DR5 was divided into low expression and high expression, and c-FLIP was divided into positive expression and negative expression. Agreement between the two evaluators was $95 \%$, and all scoring differences were solved through discussion.

Statistical analysis. All statistical analyses were performed using SPSS 24.0 (IBM Corp.). The Chi-square test was used to analyze the relationship between the expression of DR5/c-FLIP proteins and clinicopathological features of the NSCLC patients. The expression of DR5/c-FLIP protein in different tissues was statistically analyzed by Kruskal-Wallis and Bonferroni. The Spearman's rank correlation coefficient was used to assess the correlation between expression of DR5 and c-FLIP in NSCLC. Kaplan-Meier analysis was performed for overall survival curves, and statistical significance was estimated using the log-rank test. Overall survival was defined as the time from the diagnosis to the date of death or the date of last follow-up. Cox comparative hazards model was used to evaluate the independent prognostic factors of NSCLC. Two-sided statistical analysis was used and $\mathrm{P}<0.05$ was considered to be statistically significant.

\section{Results}

Expression levels of DR5 and c-FLIP proteins were significantly higher in NSCLC. Positive expression and cellular localization of DR5 and c-FLIP proteins in NSCLC and the non-cancerous control lung tissues were examined by IHC. The positive expression of DR5 protein was mainly located in 

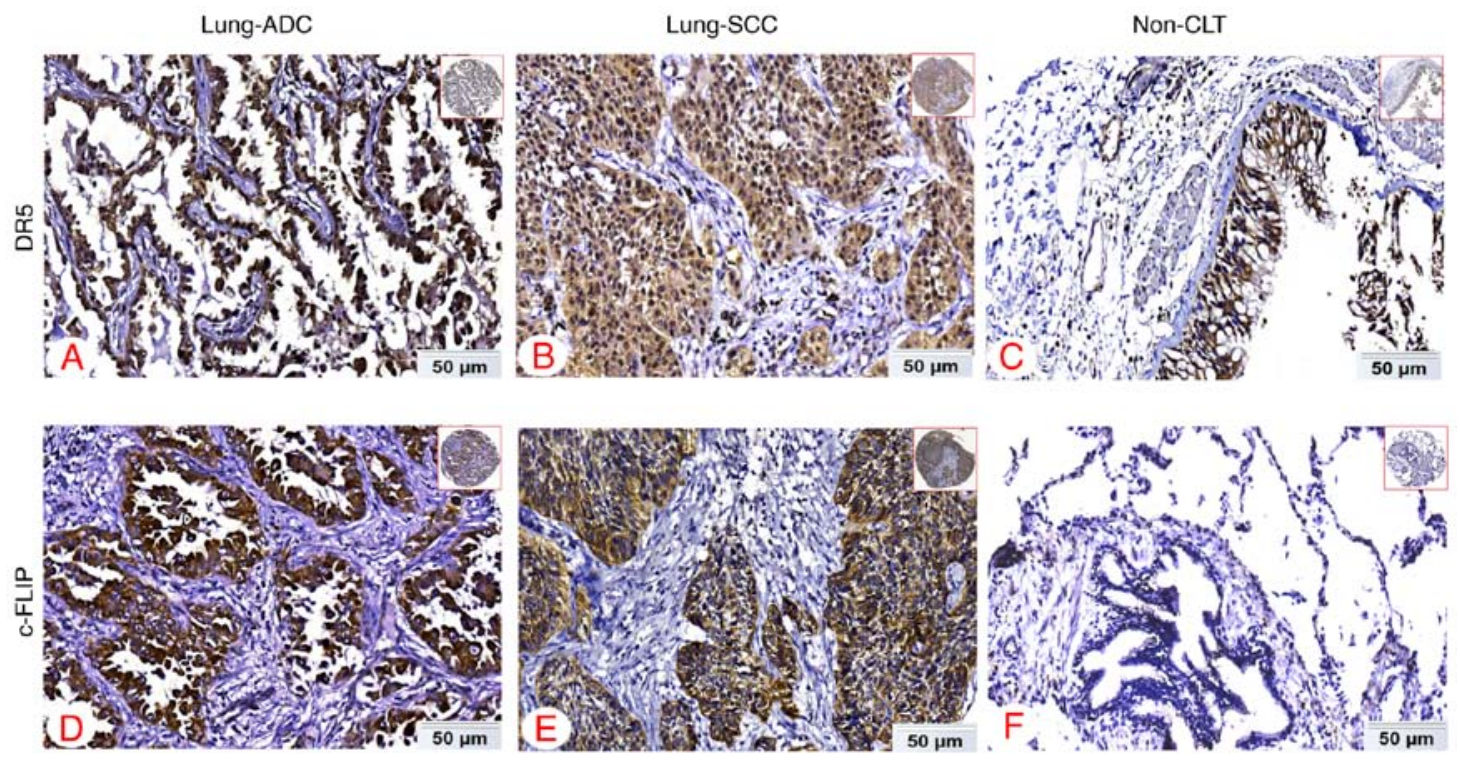

Figure 1. Expression levels of DR5 and c-FLIP proteins in lung ADC, lung SCC and Non-CLT were detected by IHC. (A and B) Strong positive staining of DR5 was observed in the cell nucleus and cytoplasm of lung ADC and SCC. (C) Positive staining of DR5 was also found in Non-CLT. (D and E) Positive staining of c-FLIP was observed in the cytoplasm of lung ADC and SCC. (F) Negative control staining of c-FLIP was found in Non-CLT. (IHC, DAB staining; original magnification $\mathrm{x} 400$ and $\mathrm{x} 50$ ). ADC, adenocarcinoma; SCC, squamous cell carcinoma; non-CLT, non-cancerous control lung tissues; IHC, immunohistochemistry.

the cell nucleus (Fig. 1A-C), and also in the cytoplasm and cell nucleus. Positive expression of c-FLIP protein was identified in the cytoplasm (Fig. 1D and E); a few cases showed positive staining both in the cytoplasm and membrane. The matched IgG isotype antibody showed no positive staining of c-FLIP as a negative control (Fig. 1F).

The percentage of high expression of DR5 protein was $12.6 \%$ (13/103), 34.7\% (43/124) and 2.0\% (1/49) in lung SCC, lung ADC and non-cancerous control lung tissues, respectively. In terms of c-FLIP protein, the percentage of positive expression was $79.6 \%$ (82/103), $51.6 \%$ (64/124) and $20.4 \%$ (10/49) in lung SCC, lung ADC and non-cancerous lung tissues. The results showed that the expression levels of DR5 and c-FLIP proteins were significantly higher in lung SCC and ADC tissues compared with levels in the control tissues $(\mathrm{P}<0.05)$ (Fig. 2).

Association between expression of DR5 and c-FLIP proteins and clinicopathological features of the NSCLC cases. We further investigated the associations between the overexpression of DR5 and c-FLIP proteins and the clinicopathological features of the NSCLC patients in a univariate Chi-square test, including age, sex, histological type, pathological degree, clinical stage, LNM (lymph node metastasis) status and smoking status (Table I). The results indicated that patients with ADC had significantly increased expression of DR5 protein than patients with SCC $(\mathrm{P}<0.001)$. However, the opposite trend was observed in regards to c-FLIP protein $(\mathrm{P}<0.001)$. Compared to clinical stage III, the percentage of cases with positive expression of c-FLIP protein was significantly lower in patients with clinical stage I and II $(\mathrm{P}=0.016)$ and younger nonsmoker patients $(\mathrm{P}=0.028, \mathrm{P}=0.019$, respectively). No differences were observed between the expression of DR5 and c-FLIP proteins and other clinicopathological features, such as sex, pathological degree and LNM status of NSCLC patients (all P>0.05). We

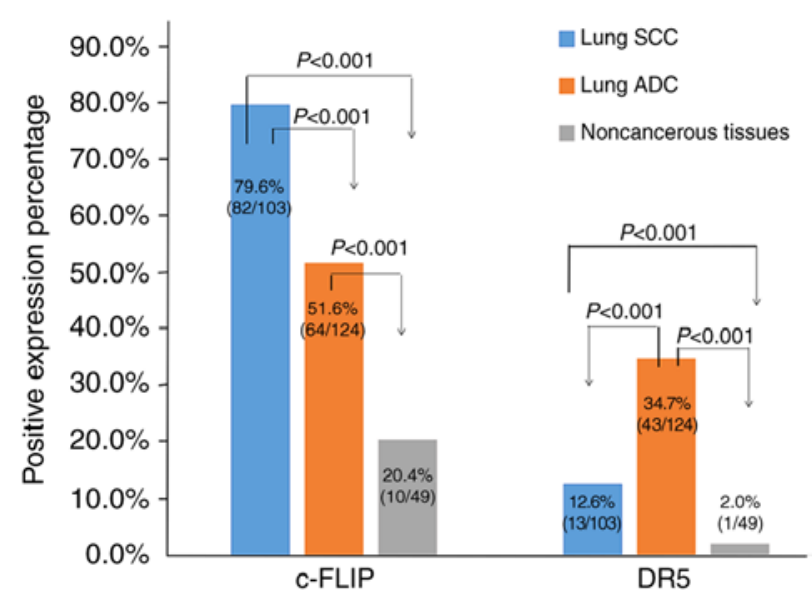

Figure 2. Comparison of the expression of DR5 and c-FLIP in lung SCC and lung $\mathrm{ADC}$ compared to the noncancerous tissues. The percentages of positive expression of DR5 in the lung SCC and lung ADC were significantly higher than these in the noncancerous tissues (both $\mathrm{P}<0.001$ ). The percentages of positive expression of c-FLIP in lung SCC and lung ADC were significantly higher than these in the noncancerous tissues (both $\mathrm{P}<0.001$ ). Positive expression of DR5 exhibited a higher percentage in lung ADC than SCC and positive expression of c-FLIP had a higher percentage in lung SCC compared to ADC, and the differences were statistically significant (both $\mathrm{P}<0.001$ ). ADC, adenocarcinoma; SCC, squamous cell carcinoma.

also investigated the relationship between the overexpression of DR5 and c-FLIP proteins and clinicopathological features of lung ADC and lung SCC, respectively (Table SII). In the lung ADC, the percentage of cases with positive expression of c-FLIP protein was significantly higher in older patients $(\mathrm{P}=0.007)$. Lung SCC patients with well/moderate differentiation had significantly increased expression of c-FLIP protein when compared with patients with poor differentiation $(\mathrm{P}=0.003)$. There was a positive correlation between positive expression of c-FLIP protein and clinical stage both in lung 
Table I. Association between expression of DR5 and c-FLIP proteins and clinicopathological features of the NSCLC patients $(\mathrm{n}=227)$.

\begin{tabular}{|c|c|c|c|c|c|c|}
\hline \multirow[b]{2}{*}{ Clinicopathological features } & \multicolumn{3}{|c|}{ DR5 } & \multicolumn{3}{|c|}{ c-FLIP } \\
\hline & High (\%) & Low $(\%)$ & P-value & Positive (\%) & Negative (\%) & P-value \\
\hline \multicolumn{7}{|l|}{ Age (years) } \\
\hline$<56(\mathrm{n}=104)$ & $30(28.8)$ & $74(71.2)$ & 0.180 & $59(56.7)$ & $45(43.3)$ & $0.028^{\mathrm{a}}$ \\
\hline$\geq 56(\mathrm{n}=123)$ & $26(21.1)$ & $97(78.9)$ & & $87(70.7)$ & $36(29.3)$ & \\
\hline \multicolumn{7}{|l|}{ Sex } \\
\hline Female $(n=63)$ & $16(25.4)$ & 47 (74.6) & 0.875 & $36(57.1)$ & $27(42.9)$ & 0.162 \\
\hline Male $(\mathrm{n}=164)$ & $40(24.4)$ & $124(75.6)$ & & $110(67.1)$ & $54(32.9)$ & \\
\hline \multicolumn{7}{|l|}{ Histological type } \\
\hline $\operatorname{ADC}(n=124)$ & $43(34.7)$ & $81(65.3)$ & $<0.001^{\mathrm{a}}$ & $64(51.6)$ & $60(48.4)$ & $<0.001^{\mathrm{a}}$ \\
\hline $\operatorname{SCC}(n=103)$ & $13(12.6)$ & $90(87.4)$ & & $82(79.6)$ & $21(20.4)$ & \\
\hline \multicolumn{7}{|l|}{ Pathological degree } \\
\hline Poor $(n=115)$ & $30(26.1)$ & $85(73.9)$ & 0.616 & $70(60.9)$ & $45(39.1)$ & 0.272 \\
\hline Well/moderate $(n=112)$ & $26(23.2)$ & $86(76.8)$ & & $76(67.9)$ & $36(32.1)$ & \\
\hline \multicolumn{7}{|l|}{ Clinical stage } \\
\hline Stage I and II $(\mathrm{n}=113)$ & $27(23.9)$ & $86(76.1)$ & 0.787 & 64 (56.6) & 49 (43.4) & $0.016^{\mathrm{a}}$ \\
\hline Stage III $(n=114)$ & $29(25.4)$ & $85(74.6)$ & & $82(71.9)$ & $32(28.1)$ & \\
\hline \multicolumn{7}{|l|}{ LNM status } \\
\hline LNM (n=127) & $30(23.6)$ & $97(76.4)$ & 0.680 & $82(64.6)$ & $45(35.4)$ & 0.929 \\
\hline No LNM $(\mathrm{n}=100)$ & $26(26.0)$ & $74(74.0)$ & & $64(64.0)$ & $36(36.0)$ & \\
\hline \multicolumn{7}{|l|}{ Smoking status } \\
\hline Smoker $(n=138)$ & $32(23.2)$ & $106(76.8)$ & 0.519 & $97(70.3)$ & $41(29.7)$ & $0.019^{\mathrm{a}}$ \\
\hline Nonsmoker $(\mathrm{n}=89)$ & $24(27.0)$ & $65(73.0)$ & & $49(55.1)$ & $40(44.9)$ & \\
\hline
\end{tabular}

The average age of all patients with NSCLC was 55.8 \pm 8.87 years. ${ }^{a} \mathrm{P}<0.05$. NSCLC, non-small cell lung cancer; ADC, adenocarcinoma; SCC, squamous cell carcinoma; LNM, lymph node metastasis.

ADC and SCC, but no significant differences were achieved $(\mathrm{P}=0.072 ; \mathrm{P}=0.078$, respectively).

Pairwise association between expression of DR5 and c-FLIP proteins in NSCLC. The pairwise association between overexpression of DR5 and c-FLIP proteins in NSCLC, including lung SCC and lung ADC, was revealed in Table II. Data showed that the high expression of DR5 protein was negatively correlated with the expression of c-FLIP protein in lung SCC $(r=-0.243, P=0.013)$. But no correlation between DR5 and c-FLIP was found in lung ADC ( $\mathrm{r}=0.027, \mathrm{P}=0.763)$.

Impact of expression of DR5 and c-FLIP proteins on the prognosis of patients with NSCLC. To further examine the impact of increased expression of DR5 and c-FLIP proteins on the OS rate of NSCLC patients, we used the Kaplan-Meier analysis to plot the survival curve and statistical significance was assessed using the log-rank test. The cumulative survival rate was $96.9 \%$ at 6 months, $88.9 \%$ at 12 months, $62.1 \%$ at 36 months and $51.7 \%$ at 60 months. The OS rate was significantly higher for NSCLC patients with high expression of DR5 protein than these with low expression ( $\mathrm{P}=0.029$, Fig. $3 \mathrm{~A})$; however, patients with positive expression of c-FLIP protein had a lower OS rate than those with negative expression $(\mathrm{P}=0.038$, Fig. $3 \mathrm{~B})$. In addition, compared with NSCLC patients with lymph node metastasis, a higher OS rate was observed for those without lymph node metastasis ( $\mathrm{P}=0.001$, Fig. $3 \mathrm{C}$ ). Of note, we also found that NSCLC patients with well and moderate differentiation had a higher OS rate than those with poor differentiation, whereas, the OS rate was lower for patients with late-stage (stage III) than those with early-stage (stage I and II) disease $(\mathrm{P}=0.002$, Fig. 3D; $\mathrm{P}<0.001$, Fig. 3E, respectively). In addition, there was no significantly prognostic impact on age, sex and histological type (Fig. 3F) (all P>0.05). Considering the differences in the expression of DR5 and c-FLIP protein in lung ADC and lung SCC, Kaplan-Meier survival analysis of these two proteins in lung SCC and ADC, respectively, is shown in Fig. S1. In lung ADC, patients with high expression of DR5 protein had prolonged survival $(\mathrm{P}=0.024)$, and patients with negative expression of c-FLIP also exhibited prolonged survival, which had weak significance $(\mathrm{P}=0.061)$, while in lung SCC, DR5 and c-FLIP proteins had no significantly prognostic impact (both $\mathrm{P}>0.05)$. Multivariate analysis using Cox proportional hazard regression was also carried out to further confirm whether the overexpression of DR5 and c-FLIP proteins was the independent prognostic factor for NSCLC patients (Table III). Data showed that high expression of DR5 protein and negative expression of c-FLIP protein may serve as independent 
Table II. Pairwise association between expression of DR5 and c-FLIP proteins in lung SCC and ADC.

\begin{tabular}{lcccc}
\hline & \multicolumn{2}{c}{ ADC } & & SCC \\
\cline { 2 - 5 } & DR5 & c-FLIP & DR5 & c-FLIP \\
\hline DR5 & & & \\
Spearman's correlation coefficient & 1 & 0.027 & 1 & -0.243 \\
Sig. (2-tailed) & & 0.763 & $0.013^{\text {a }}$ \\
\hline
\end{tabular}

Values are expressed as Spearman's correlation coefficients. ${ }^{\mathrm{P}}<0.05$. ADC, adenocarcinoma; SCC, squamous cell carcinoma.

A

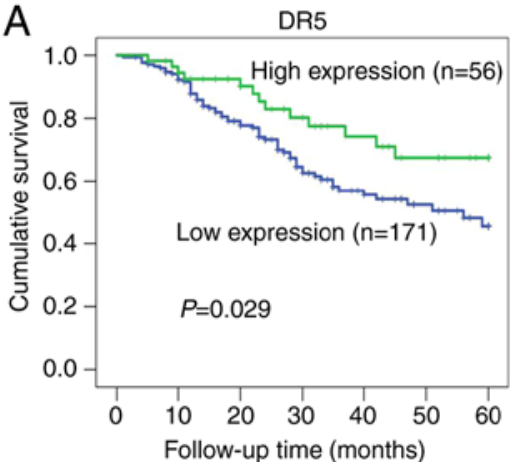

D

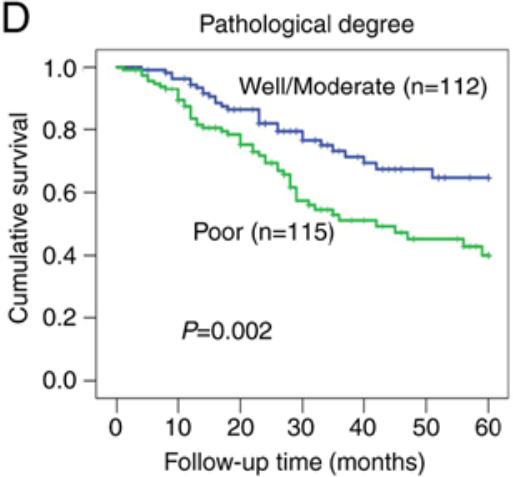

B

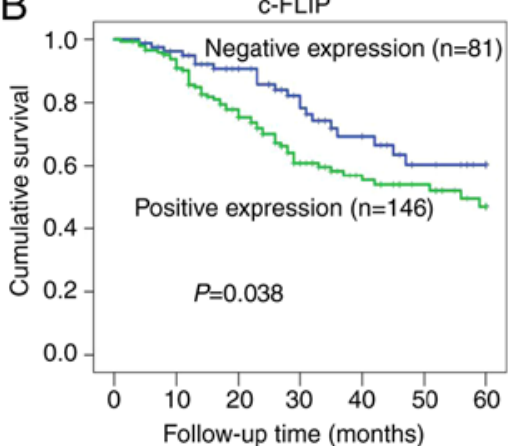

E

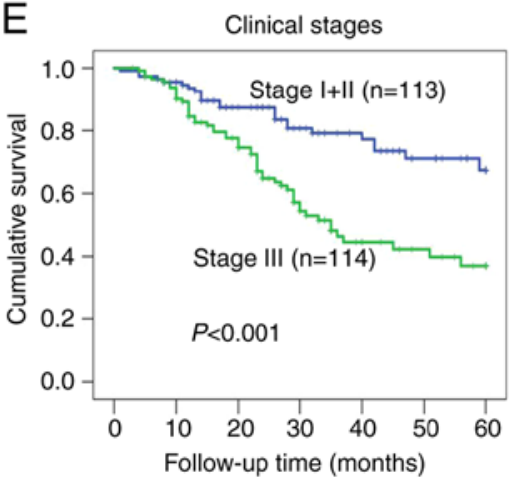

C

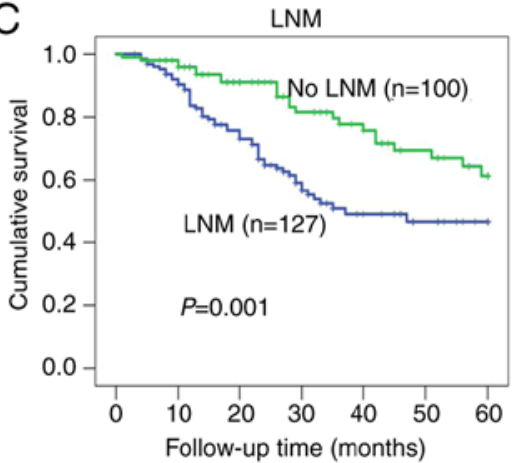

F

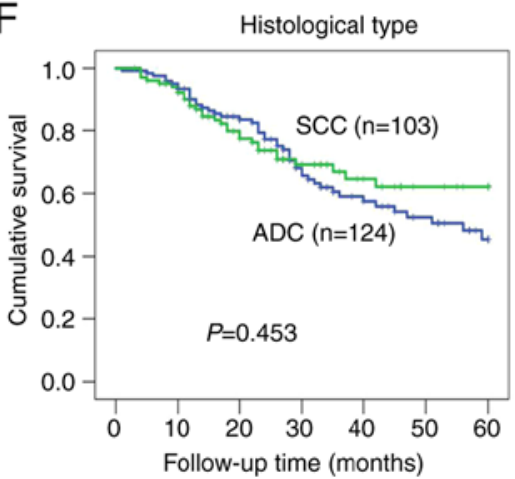

Figure 3. Kaplan-Meier curves for overall survival of NSCLC patients as assessed using the log-rank test (all tests were 2-sided). (A) High expression of DR5 was significantly associated with a prolonged overall survival $(\mathrm{P}=0.029)$. (B) Negative expression of c-FLIP was significantly associated with a more favorable prognosis $(\mathrm{P}=0.046)$, as well as $(\mathrm{C})$ without lymph node metastasis $(\mathrm{P}=0.001)$, (D) well and moderate differentiation $(\mathrm{P}=0.002)$ and $(\mathrm{E})$ early-stage disease ( $\mathrm{P}<0.001)$. (F) Histological type was not significantly associated with overall survival $(\mathrm{P}=0.453)$. NSCLC, non-small cell lung cancer.

better prognostic factors for NSCLC patients $(\mathrm{P}=0.016$ $\mathrm{P}=0.035$, respectively), as well as early-stage $(\mathrm{P}=0.013)$, well and moderate differentiation $(\mathrm{P}=0.011)$ and without lymph node metastasis $(\mathrm{P}=0.022)$. No clinical prognostic effect was detected in regards to histological type, age and sex of NSCLC (all $\mathrm{P}>0.05$ ).

\section{Discussion}

Apoptosis is an active suicide process regulated by gene coding in the process of growth, differentiation, development and pathology, also known as programmed cell death $(7,25)$ and mainly includes intrinsic and extrinsic pathways. Although apoptosis plays a critical role in eliminating damaged cells, abnormal regulation of apoptosis can lead to imbalance between cell proliferation and cell death, leading to tumorigenesis and drug resistance (26). We conducted this study to examine the relationship between DR5/c-FLIP protein and clinicopathological/prognostic implications in NSCLC patients treated with chemotherapy after radical resection. Leithner et al found that TRAIL receptors were not only observed in the cytoplasm of NSCLC cells, but also in the nucleus, which was further confirmed in our study (27). We demonstrated that the expression levels of DR5 and c-FLIP protein were higher in lung SCC and ADC, compared with levels in the non-cancerous tissues, which was consistent with previous reports $(28,29)$. DR5 and c-FLIP proteins were also found to be expressed at higher levels than their normal counterparts in a range of other tumors, including colon, cervix and breast (30-34), which indicates they may play an important role in various cancers. What's more, our data also showed that DR5 expression was significantly increased in lung ADC compared to SCC, while 
Table III. Summary of the univariate and multivariate analyses for overall survival in the 227 NSCLC cases.

\begin{tabular}{|c|c|c|c|c|c|c|}
\hline \multirow[b]{2}{*}{ Variables } & \multicolumn{3}{|c|}{ Univariate analysis } & \multicolumn{3}{|c|}{ Multivariate analysis } \\
\hline & Average survival time (SE) & $95 \% \mathrm{CI}$ & P-value & $\operatorname{Exp}(\beta)$ & $95 \% \mathrm{CI}$ & P-value \\
\hline \multicolumn{7}{|l|}{ DR5 } \\
\hline High expression & $49.02(2.63)$ & $(43.87,54.18)$ & $0.029^{\mathrm{a}}$ & 2.152 & $(1.155,4.009)$ & $0.016^{\mathrm{a}}$ \\
\hline Low expression & $41.71(1.76)$ & $(38.26,45.15)$ & & & & \\
\hline \multicolumn{7}{|l|}{ c-FLIP } \\
\hline Positive expression & $41.40(1.92)$ & $(37.64,45.16)$ & $0.038^{\mathrm{a}}$ & 0.558 & $(0.325,0.958)$ & $0.035^{\mathrm{a}}$ \\
\hline Negative expression & $47.48(2.26)$ & $(43.06,51.91)$ & & & & \\
\hline \multicolumn{7}{|l|}{ Clinical stage } \\
\hline Stage I and II & $49.73(1.88)$ & $(46.06,53.41)$ & $0.000^{\mathrm{a}}$ & 0.517 & $(0.307,0.870)$ & $0.013^{\mathrm{a}}$ \\
\hline Stage III & $37.81(2.12)$ & $(33.67,41.96)$ & & & & \\
\hline \multicolumn{7}{|l|}{ LNM status } \\
\hline LNM & $39.10(2.07)$ & $(35.03,43.16)$ & $0.001^{\mathrm{a}}$ & 1.839 & $(1.092,3.097)$ & $0.022^{\mathrm{a}}$ \\
\hline No LNM & $49.75(1.92)$ & $(45.99,53.51)$ & & & & \\
\hline \multicolumn{7}{|l|}{ Pathological degree } \\
\hline Well and moderate & $47.91(1.93)$ & $(44.12,51.70)$ & $0.002^{*}$ & 0.540 & $(0.335,0.870)$ & $0.011^{\mathrm{a}}$ \\
\hline Poor & $39.24(2.18)$ & $(34.97,43.51)$ & & & & \\
\hline \multicolumn{7}{|l|}{ Histological type } \\
\hline $\mathrm{ADC}$ & $42.86(1.94)$ & $(39.05,46.67)$ & 0.453 & 1.685 & $(0.998,2.845)$ & 0.051 \\
\hline $\mathrm{SCC}$ & $44.78(2.28)$ & $(40.32,49.25)$ & & & & \\
\hline \multicolumn{7}{|l|}{ Sex } \\
\hline Female & $45.23(2.56)$ & $(40.22,50.24)$ & 0.613 & 0.656 & $(0.385,1.117)$ & 0.121 \\
\hline Male & $42.86(1.82)$ & $(39.29,46.43)$ & & & & \\
\hline \multicolumn{7}{|l|}{ Age (years) } \\
\hline$<56$ & $44.25(2.23)$ & $(39.89,48.61)$ & 0.574 & 1.061 & $(0.664,1.693)$ & 0.805 \\
\hline$\geq 56$ & $42.88(2.01)$ & $(38.94,46.82)$ & & & & \\
\hline
\end{tabular}

NSCLC, non-small cell lung cancer; ADC, adenocarcinoma; SCC, squamous cell carcinoma; LN, lymph node; LNM, lymph node metastasis; $\mathrm{CI}$, confidence interval; SE, standard error; $\operatorname{Exp}(\beta)$, odds ratio. ${ }^{\mathrm{a}} \mathrm{P}<0.05$.

the expression of c-FLIP was higher in lung SCC than ADC, and late-stage patients had higher c-FLIP than early-stage patients, which needs to be further investigated. The results demonstrated that the expression of DR5 and c-FLIP proteins varied according to clinicopathological features which showed predictive value for the expression of these two proteins.

In various studies, the prognostic significance of DR5 protein expression in tumors has been determined with conflicting results, and the results are different even in the same tumor type. Decreased expression of DR5 was found to be associated with progression in melanoma (35), while DR5 expression was found to be related to poor survival in breast cancer (32). In NSCLC, the clinical outcomes are different. Spierings et al reported that DR5 expression increased the risk of death but the statistical significance was weak (36). However, in another study, the researchers demonstrated that both nuclear and cytoplasmic DR5 were correlated with improved survival in advanced NSCLC patients (27). The difference in prognostic value of DR5 may be related to the different types of tumors and the different treatments received by the patients. In the present study, all patients received platinum-based chemotherapy after radical resection. The results showed that the patients with high expression of DR5 had a significantly longer overall survival than these with low expression and the difference was statistically significant. Moreover, DR5 protein was also a favorable independent prognostic factor in lung ADC patients, but no significance was achieved in lung SCC patients. Our findings were in line with previous research (27) and further indicated that DR5 protein may be used as a predictor for tumor response to chemotherapy, which warrants further in-depth experiments for confirmation.

The c-FLIP protein is a potent anti-apoptotic protein that is overexpressed in many malignant tumors, and facilitates cancer cell escape from apoptosis (37). Recently, evidence has emerged that the c-FLIP protein is the main reason for resistance to chemotherapeutic drugs. Kim et al reported that overexpression of c-FLIPs inhibited oxaliplatin-mediated apoptosis by enhancing XIAP stability and activating the Akt pathway in human renal cancer cells (38). Another research revealed that colon cancer cells were resistant to oxaliplatin due to increased c-FLIP and Mcl-1 proteins (myeloid cell leukemia-1, a new member of the $\mathrm{Bcl}-2$ family), resulting in 
the upregulation of $\mathrm{NF}-\kappa \mathrm{B}$, which ultimately led to anti-apoptotic capacity (39). In the present study, we found that the overexpression of c-FLIP protein acts as a poor independent prognostic biomarker for NSCLC patients.

To the best of our knowledge, this was the first study to examine the prognostic value of DR5 and c-FLIP proteins in NSCLC patients, including lung ADC and lung SCC patients. Our results showed that DR5 is a favorable prognostic factor while c-FLIP is a worse prognostic factor. DR5 was negatively correlated with c-FLIP in lung SCC specimens, which warrants further in-depth experiments to further confirm these findings. In the present study, all patients underwent similar treatment (lobectomy + LN dissection and adjuvant platinum doublet chemotherapy) and baseline characteristics, thus we can easily conclude that DR5 and c-FLIP may serve as novel prognostic biomarkers for these NSCLC patients. To further assess the importance of DR5 and c-FLIP proteins as prognostic factors for NSCLC patients treated with chemotherapy, a larger number of studies are needed and more detailed patient characteristics will be collected in future studies. In addition, a good response to chemotherapy may be an important factor in prolonging the survival of NSCLC patients and a good prognosis may indicate a good response to chemotherapy. It has been shown that DR5/c-FLIP proteins are related to drug-induced apoptosis $(5,37,38)$, thus DR5/c-FLIP proteins may be used as predictive markers for the response of possible residual tumors to adjuvant chemotherapy, which needs more in-depth experiments to further confirm. In addition, disease (or relapse)-free survival is another important endpoint, but this was a retrospective study and was followed up by telephone interview which made the follow-up and imaging evaluation difficult to ensure balance and the evaluation was prone to deviation. Thus, it was difficult to obtain the accurate disease (or relapse)-free survival. In future research, we will give added attention to the standard follow-up method and disease (or relapse)-free survival.

In conclusion, our findings strongly suggest that high expression of DR5 and loss of expression of c-FLIP may be promising favorable independent prognostic markers for NSCLC patients. What's more, DR5 and c-FLIP proteins could also be used as predictors for the tumor response to chemotherapy drugs and provide a simply strategy to select patients for this therapy, but the prognostic value of DR5/c-FLIP proteins in NSCLC patients treated with surgical resection and chemotherapy is the more important role.

\section{Acknowledgements}

Not applicable.

\section{Funding}

The present study was supported by grants from The National Natural Sciences Foundations of China (nos. 81773218, 81703009 and 81472773) and The Natural Sciences Foundations of Hunan Province (no. 2017JJ3457).

\section{Availability of data and materials}

All data generated or analyzed during this study are included in this published article.

\section{Authors' contributions}

SF and HZ conceived and designed the study. HZ, YZhang, SL and JL performed the experiments. $\mathrm{HZ}$ and SF wrote the paper. SF, QW, YZhan and SL reviewed and edited the manuscript. All authors read and approved the manuscript and agree to be accountable for all aspects of the research in ensuring that the accuracy or integrity of any part of the work are appropriately investigated and resolved.

\section{Ethics approval and consent to participate}

All procedures used in this study were approved by the Ethics Committee of The Second Xiangya Hospital of Central South University (Scientific and Research Ethics Committee, no. S039/2011) and any related procedures were performed in accordance with relevant guidelines and regulations. Written informed consent was obtained from all patients.

\section{Patient consent for publication}

Not applicable.

\section{Competing interests}

The authors declare that they have no competing interests.

\section{References}

1. Siegel RL, Miller KD and Jemal A: Cancer statistics, 2017. CA Cancer J Clin 67: 7-30, 2017.

2. Zheng H, Zhan Y, Liu S, Lu J, Luo J, Feng J and Fan S: The roles of tumor-derived exosomes in non-small cell lung cancer and their clinical implications. J Exp Clin Cancer Res 37: 226, 2018.

3. Little AG, Gay EG, Gaspar LE and Stewart AK: National survey of non-small cell lung cancer in the United States: Epidemiology, pathology and patterns of care. Lung Cancer 57: 253-260, 2007.

4. Gadgeel SM, Stevenson JP, Langer CJ, Gandhi L, Borghaei H, Patnaik A, Villaruz LC, Gubens M, Hauke R, Yang JC, et al: Pembrolizumab and platinum-based chemotherapy as first-line therapy for advanced non-small-cell lung cancer: Phase 1 cohorts from the KEYNOTE-021 study. Lung Cancer 125: 273-281, 2018.

5. Han JY, Hong EK, Choi BG, Park JN, Kim KW, Kang JH, Jin JY, Park SY, Hong YS and Lee KS: Death receptor 5 and Bcl-2 protein expression as predictors of tumor response to gemcitabine and cisplatin in patients with advanced non-small-cell lung cancer. Med Oncol 20: 355-362, 2003.

6. Houghton JA: Apoptosis and drug response. Curr Opin Oncol 11: 475-481, 1999.

7. Hengartner MO: The biochemistry of apoptosis. Nature 407: 770-776, 2000.

8. Walczak H, Degli-Esposti MA, Johnson RS, Smolak PJ, Waugh JY, Boiani N, Timour MS, Gerhart MJ, Schooley KA, Smith CA, et al: TRAIL-R2: A novel apoptosis-mediating receptor for TRAIL. EMBO J 16: 5386-5397, 1997.

9. Sheridan JP, Marsters SA, Pitti RM, Gurney A, Skubatch M, Baldwin D, Ramakrishnan L, Gray CL, Baker K, Wood WI, et al: Control of TRAIL-induced apoptosis by a family of signaling and decoy receptors. Science 277: 818-821, 1997.

10. Chinnaiyan AM, O'Rourke K, Tewari M and Dixit VM: FADD, a novel death domain-containing protein, interacts with the death domain of fas and initiates apoptosis. Cell 81: 505-512, 1995.

11. Bodmer JL, Holler N, Reynard S, Vinciguerra P, Schneider P, Juo P, Blenis J and Tschopp J: TRAIL receptor-2 signals apoptosis through FADD and caspase-8. Nat Cell Biol 2: 241-243, 2000.

12. Ashkenazi A: Targeting the extrinsic apoptosis pathway in cancer. Cytokine Growth Factor Rev 19: 325-331, 2008. 
13. Irmler M, Thome M, Hahne M, Schneider P, Hofmann K, Steiner V, Bodmer JL, Schröter M, Burns K, Mattmann C, et al: Inhibition of death receptor signals by cellular FLIP. Nature 388: 190-195, 1997.

14. Li FY, Jeffrey PD, Yu JW and Shi Y: Crystal structure of a viral FLIP: Insights into FLIP-mediated inhibition of death receptor signaling. J Biol Chem 281: 2960-2968, 2006.

15. Yang JK: FLIP as an anti-cancer therapeutic target. Yonsei Med J 49: 19-27, 2008.

16. Golks A, Brenner D, Fritsch C, Krammer PH and Lavrik IN: c-FLIPR, a new regulator of death receptor-induced apoptosis. J Biol Chem 280: 14507-14513, 2005.

17. French LE and Tschopp J: Defective death receptor signaling as a cause of tumor immune escape. Semin Cancer Biol 12: 51-55, 2002.

18. Hanahan D and Weinberg RA: The hallmarks of cancer. Cell 100: 57-70, 2000.

19. Travis WD, Brambilla E, Noguchi M, Nicholson AG, Geisinger KR, Yatabe Y, Beer DG, Powell CA, Riely GJ, Van Schil PE, et al: International association for the study of lung cancer/American thoracic society/European respiratory society international multidisciplinary classification of lung adenocarcinoma. J Thorac Oncol 6: 244-285, 2011

20. Detterbeck FC, Boffa DJ, Kim AW and Tanoue LT: The eighth edition lung cancer stage classification. Chest 151: 193-203, 2017.

21. Wen Q, Wang W, Luo J, Chu S, Chen L, Xu L, Zang H, Alnemah MM, Ma J and Fan S: CGP57380 enhances efficacy of RAD001 in non-small cell lung cancer through abrogating mTOR inhibition-induced phosphorylation of eIF4E and activating mitochondrial apoptotic pathway. Oncotarget 7: 27787-27801, 2016

22. Fan SQ, Ma J, Zhou J, Xiong W, Xiao BY, Zhang WL, Tan C, Li XL, Shen SR, Zhou M, et al: Differential expression of Epstein-Barr virus-encoded RNA and several tumor-related genes in various types of nasopharyngeal epithelial lesions and nasopharyngeal carcinoma using tissue microarray analysis. Hum Pathol 37: 593-605, 2006.

23. Li J, Wen Q, Xu L, Wang W, Luo J, Chu S, Xie G, Shi L, Huang D, Li J and Fan S: Fatty acid synthase-associated protein with death domain: A prognostic factor for survival in patients with nasopharyngeal carcinoma. Hum Pathol 45: 2447-2452, 2014.

24. Zheng J, Li J, Xu L, Xie G, Wen Q, Luo J, Li D, Huang D and Fan S: Phosphorylated Mnk1 and eIF4E are associated with lymph node metastasis and poor prognosis of nasopharyngeal carcinoma. PLoS One 9: e89220, 2014.

25. Elmore S: Apoptosis: A review of programmed cell death. Toxicol Pathol 35: 495-516, 2007.

26. Johnstone RW, Ruefli AA and Lowe SW: Apoptosis: A link between cancer genetics and chemotherapy. Cell 108: 153-164, 2002.

27. Leithner K, Stacher E, Wurm R, Ploner F, Quehenberger F, Wohlkoenig C, Bálint Z, Polachova J, Olschewski A, Samonigg $\mathrm{H}$, et al: Nuclear and cytoplasmic death receptor 5 as prognostic factors in patients with non-small cell lung cancer treated with chemotherapy. Lung Cancer 65: 98-104, 2009.

28. Daniels RA, Turley H, Kimberley FC, Liu XS, Mongkolsapaya J, Ch'En P, Xu XN, Jin BQ, Pezzella F and Screaton GR: Expression of TRAIL and TRAIL receptors in normal and malignant tissues. Cell Res 15: 430-438, 2005.
29. Hutchinson RA, Coleman HG, Gately K, Young V, Nicholson S, Cummins R, Kay E, Hynes SO, Dunne PD, Senevirathne S, et al: IHC-based subcellular quantification provides new insights into prognostic relevance of FLIP and procaspase- 8 in non-small-cell lung cancer. Cell Death Discov 3: 17050, 2017.

30. Koornstra JJ, Kleibeuker JH, van Geelen CM, Rijcken FE, Hollema H, de Vries EG and de Jong S: Expression of TRAIL (TNF-related apoptosis-inducing ligand) and its receptors in normal colonic mucosa, adenomas, and carcinomas. J Pathol 200: 327-335, 2003.

31. Ozawa F, Friess H, Kleeff J, Xu ZW, Zimmermann A, Sheikh MS and Büchler MW: Effects and expression of TRAIL and its apoptosis-promoting receptors in human pancreatic cancer. Cancer Lett 163: 71-81, 2001.

32. McCarthy MM, Sznol M, DiVito KA, Camp RL, Rimm DL and Kluger HM: Evaluating the expression and prognostic value of TRAIL-R1 and TRAIL-R2 in breast cancer. Clin Cancer Res 11: 5188-5194, 2005

33. Korkolopoulou P, Saetta AA, Levidou G, Gigelou F, Lazaris A, Thymara I, Scliri M, Bousboukea K, Michalopoulos NV, Apostolikas N, et al: c-FLIP expression in colorectal carcinomas: Association with Fas/FasL expression and prognostic implications. Histopathology 51: 150-156, 2007.

34. Wang W, Wang S, Song X, Sima N, Xu X, Luo A, Chen G, Deng D, Xu Q, Meng L, et al: The relationship between c-FLIP expression and human papillomavirus E2 gene disruption in cervical carcinogenesis. Gynecol Oncol 105: 571-577, 2007.

35. Zhuang L, Lee CS, Scolyer RA, McCarthy SW, Zhang XD, Thompson JF, Screaton G and Hersey P: Progression in melanoma is associated with decreased expression of death receptors for tumor necrosis factor-related apoptosis-inducing ligand. Hum Pathol 37: 1286-1294, 2006.

36. Spierings DC, de Vries EG, Timens W, Groen HJ, Boezen HM and de Jong S: Expression of TRAIL and TRAIL death receptors in stage III non-small cell lung cancer tumors. Clin Cancer Res 9: 3397-3405, 2003.

37. Day TW, Najafi F, Wu CH and Safa AR: Cellular FLICE-like inhibitory protein (c-FLIP): A novel target for taxol-induced apoptosis. Biochem Pharmacol 71: 1551-1561, 2006.

38. Kim S, Lee TJ,Park JW and Kwon TK: Overexpression of cFLIPs inhibits oxaliplatin-mediated apoptosis through enhanced XIAP stability and Akt activation in human renal cancer cells. J Cell Biochem 105: 971-979, 2008.

39. Jani TS, DeVecchio J, Mazumdar T, Agyeman A and Houghton JA: Inhibition of NF-kappaB signaling by quinacrine is cytotoxic to human colon carcinoma cell lines and is synergistic in combination with tumor necrosis factor-related apoptosis-inducing ligand (TRAIL) or oxaliplatin. J Biol Chem 285: 19162-19172, 2010.

This work is licensed under a Creative Commons Attribution-NonCommercial-NoDerivatives 4.0 International (CC BY-NC-ND 4.0) License. 